\title{
A novel method for fast and robust estimation of fluorescence decay dynamics using constrained least-square deconvolution with Laguerre expansion
}

\author{
Jing Liu, Yang Sun, Jinyi Qi, and Laura Marcu \\ Department of Biomedical Engineering, University of California, Davis, 95616
}

\begin{abstract}
We report a novel method for estimating fluorescence impulse response function (fIRF) from noise-corrupted time-domain fluorescence measurements of biological tissue. This method is based on the use of higher-order Laguerre basis functions (LBFs) and a constrained least-square (CLS) approach that addresses the problem of overfitting due to increased model complexity. The method was extensively evaluated on fluorescence data from simulation, fluorescent standard dyes, ex vivo tissue samples of atherosclerotic plaques, and in vivo oral carcinoma. Current results demonstrate that this new method allows for rapid and accurate deconvolution of multiple channel fluorescence decays without adaptively adjusting the Laguerre scale parameter. The appropriate choice of the scale parameter is essential for accurate estimation of the fIRF. The method described here is anticipated to play an important role in the development of computational techniques for real-time analysis of time-resolved fluorescence data from biological tissues and to support the advancement of fluorescence lifetime instrumentation for biomedical diagnostics by providing a means for on-line robust analysis of fluorescence decay.
\end{abstract}

\section{Introduction}

Fluorescence lifetime spectroscopy and imaging techniques have demonstrated potential for characterization of biological tissues. Recent studies have shown that these techniques can provide useful label-free optical molecular contrast for clinical and biomedical research (Cubeddu et al., 2002; Elson et al., 2006; Galletly et al., 2008; König, 2008; Sun et al., 2009b; Marcu et al., 2009; Uehlinger et al., 2009; McGinty et al., 2010). The fluorescence decay profiles of biological tissues, however, are often complex. This is due to not only the presence of many distinct fluorophores in tissues but also a range of fluorophore environments that can affect their fluorescence lifetime (Lakowicz, 2006). Computational methods for fast and accurate analysis of fluorescence intensity decay profiles from timeresolved measurements of tissues play an important role in the advancement of these techniques as practical tools in biomedical diagnostics.

In the context of time-domain fluorescence techniques, the measured fluorescence response from biological tissues to a light excitation pulse stimulus is a convolution of fluorescence impulse response function (fIRF) representing the intrinsic tissue fluorescence with the instrument impulse response function (iIRF) representing the distorted light pulse due to instrument electronics and other delay components. Time-resolved fluorescence spectroscopy (TRFS) and fluorescence lifetime imaging (FLIM) techniques for tissue characterization require simultaneous and reliable recovery (i.e. deconvolution) of intrinsic

Correspondence to: Jing Liu; Laura Marcu.

lmarcu@ucdavis.edu; jnliu@ucdavis.edu. 
fIRFs from multiple spectral and spatial channel (e.g. wavelengths, locations) measurements.

Numerous mathematical tools have been devised for the recovery of fIRF. These include integral transform approaches, such as Fourier (Andre et al., 1979) and Laplace (Gafni et al., 1975) transforms, method of moments (Isenberg et al., 1973), method of modulating function (Valeur, 1978), Prony method (Zhang et al., 1996; Niesner et al., 2004), expectation-maximization method ( $\mathrm{Ng}$ et al., 2009), as well as least-square deconvolution (LSD) methods including both nonlinear and ordinary least-square. Many of these deconvolution techniques are most useful when the underlying fIRF are modeled as a summation of exponential decay functions (i.e. multi-exponential expansion). However, LSD is not limited to the functional form of fIRF models and has been commonly adopted when models other than multiple component exponentials are of interests (Ware et al., 1973; Murata et al., 1995; Lee et al., 2001). In addition, LSD has been shown to be reliable and robust to measurement noise in a variety of simulation and experiment settings (McKinnon et al., 1977; O'Connor et al., 1979). In general, LSD searches for fIRF that best fits to the observed data after re-convolving with iIRF in the least-square sense.

The use of multi-exponential expansion to represent the fIRF has been critically reviewed in literature (e.g. Lakowicz, 2006). For tissue characterization difficulties arise in two aspects. First, multi-exponential fIRF models are nonlinear in their parameters and therefore, leastsquare estimation of exponential expansion parameters (including expansion coefficient and decay constant from individual component) involve nonlinear optimization problems that usually have non-unique solution and is computationally expensive. Second, the number of exponential decay components used cannot be justified from a physical perspective for complex biological fluorescence systems. Except for limited situations, parameters associated with each exponential component cannot be interpreted in terms of underlying fluorophores content. As previously noted (Lee et al., 2001), many fluorescence systems may contain unlimited number of exponential decays.

Alternatively, the intrinsic fIRF can be expanded onto an ordered basis set of orthonormal functions, namely, Laguerre basis functions (LBFs), as illustrated and validated in our previous studies (Maarek et al., 2000; Jo et al., 2004; Jo et al., 2006). A finite-dimension Laguerre basis set is completely specified by two basis parameters: scale, $a$ and dimension, $L$ (i.e. the number of LBFs within the ordered basis set such that the highest order of LBFs is $L-1)$. For given $a$ and $L$, Laguerre expansions provide linear parameterization of the fIRF without assumption of its exact functional form. It should be noted that expansion of impulse response functions with LBFs has a long history in the context of system modeling and identification (Heuberger et al., 2005). It has been proven to be useful in identifying biological systems (Westwick and Kearney, 2003). A recent study (Agrawal et al., 2010) also showed superior sensitivity of Laguerre expansion based techniques in disease detection.

The practical use of Laguerre expansion of fIRF requires selection of the Laguerre basis set ( $a$ and $L$ ). However, the optimal choice of basis parameters is not trivial, since the choices of scale and dimension parameters are coupled. Marmarelis (Marmarelis, 1993) suggested selecting a set of LBFs that have "significant" coverage of fIRF and that diminish not long after fIRF decays to zero. However, this heuristic criterion is often hard to implement when fIRF consists of complex decay dynamics including a broad range of decays from fast (subnanosecond) to slow (several nanoseconds) components.

Recently, several groups chose to treat the scale parameter as an optimization parameter and automated its search as part of the LSD (Lee et al., 1994; McCombie et al., 2005; Boukis et 
al., 2006; Dankers and Westwick, 2006; Dabir et al., 2009). The dimension parameter was selected based on subsequent analysis on information-theoretic metrics, such as Minimal Description Length (Dabir et al., 2009), or Akaike Information Criteria (Lee et al., 1994). Alternatively, the selection of $a$ could also be based on information-theoretic metrics (Yuan et al., 1999) or other sub-optimal criteria (Fu and Dumont, 1993; Tanguy et al., 1995; Dankers and Westwick, 2010). These approaches to select basis parameters are nonlinear in nature. Numerically, LSD using Laguerre expansion of fIRF with automatic basis parameters selection is similar to that using multi-exponential expansion. Iterative nonlinear optimizations are required, which are often challenging computationally. Moreover, for TRFS and FLIM applications, when near real-time deconvolution of measurements from multiple wavelengths/pixels is required, it is impractical to select Laguerre basis set through nonlinear optimization for individual measurement. A recent work addressed this problem for FLIM application (Pande and Jo, 2011), where a unique $a$ value was selected for all pixel measurements by minimizing a global cost function. Obviously, trade-off has been made for deconvolution across pixels.

In general, high dimensional Laguerre basis set (i.e. large number of LBFs) is preferred for identification of systems with complex fIRF where a broad range of decays is present; higher the number of LBFs larger the degree of freedom available to capture the characteristics of a system response with complex decay dynamics. It was also observed that by incorporating more LBFs, the LSD become less sensitive to the choice of scale parameter (Dankers and Westwick, 2006). These facts suggest that it is possible to use one Laguerre basis set with pre-specified $a$ and $L$ values for deconvolving fIRF with a broad range of decay dynamics, when many LBFs are used. Previous implementation of LSD with Laguerre expansion, however, relied on the ordinary least-square (OLS) method. Including too many LBFs (or too many degrees of freedom) often induces "overfitting" (i.e. fitting to the noise) for measurements with low signal-to-noise ratio (SNR) and results in physically unrealistic fIRFs.

The main goals of this study are to a) Develop a constrained least-square (CLS) approach for the deconvolution of fIRF using high-order LBFs. This has the potential to overcome the limitation of the OLS method since it is insensitive to measurement noise especially when high-dimensional Laguerre expansion of fIRF is used; b) Introduce a technique for choosing the appropriate combination of $a$ and $L$, suitable for fast deconvolution of fluorescence decays; and c) Validate the accuracy of this new approach on simulated TRFS and FLIM data and experimental data from TRFS and FLIM measurements of standard fluorescence dyes and tissue samples.

\section{Theory}

Both the fluorescence system (tissue) and the instrument system are assumed to be linear time invariant systems, such that they are entirely characterized by their impulse response functions. The measured signal as a function of time, $y(t)$ is the convolution of fIRF, $h(t)$ with iIRF, $I(t)$. In discrete time representation, for $N$ equal sampling time points $t_{i}=(i-$ 1) $\delta t, i=1, \ldots, N$ and sampling interval, $\delta t$, we have,

$$
y(k)=\sum_{i=1}^{k} I(k-i) \cdot h(i)+\varepsilon_{k}
$$

where additive white noise (at time point $t_{k}$ ), $\varepsilon_{k}$ for $k=1, \ldots, N$ is assumed. We are interested in estimating fIRF, $h(k)$ from measured iIRF and time-resolved fluorescence signals. 
In general, fIRF, $h(k)$ can be parameterized in various ways. For example, expansion of $h(k)$ onto multi-exponential components is a common strategy in analyzing time-resolved fluorescence measurement. A stretched-exponential function was also used for its more accurate description of underlying physical dynamics (Lee et al., 2001). The choice of parameterization is mainly based on considerations of physical reality and numerical simplicity. In other words, a good parameterization should accurately represent the relaxation dynamics of the system while being efficient in the numerical evaluation of such dynamics.

In previous work, we expanded $h(k)$ onto an ordered set of discrete time LBFs, $b_{\Lambda}(k, a)$,

$$
h(k)=\sum_{l=0}^{L-1} c_{l} b_{l}(k ; \alpha)
$$

where $L$ and $a$ are the basis parameters and $c_{l}$ is the $t^{\text {th }}$ expansion coefficient. The $t^{\text {th }}$ discrete time LBF is defined as,

$$
b_{l}(k ; \alpha)=\alpha^{(k-l) / 2}(1-\alpha)^{1 / 2} \sum_{i=0}^{l}(-1)^{i}\left(\begin{array}{c}
k \\
i
\end{array}\right)\left(\begin{array}{c}
l \\
i
\end{array}\right) \alpha^{l-i}(1-\alpha)^{i}
$$

for $I=0, \ldots, L-1$ and $0<a<1$. It is well known that LBFs form an orthonormal basis set such that,

$$
\boldsymbol{b}_{l}^{T} \boldsymbol{b}_{l^{\prime}}=\delta_{l l^{\prime}}
$$

where $\boldsymbol{b}_{I}=\left[b_{I}(1 ; a), b_{I}(2 ; a), \ldots, b_{I}(k ; a), \ldots, b_{I}(N ; a)\right]^{T}$ and $\delta_{I I}$ denotes Kronecker delta function. Note that the exact equality (4) holds only for $N \rightarrow \infty$, and for finite $N b_{l}^{T} \boldsymbol{b}_{l^{\prime}} \approx \delta_{l l^{\prime}}$. For a set of $L$ basis functions, define $B=\left[\boldsymbol{b}_{0}, \mathbf{b}_{1}, \ldots, \mathbf{b}_{\mathrm{L}-1}\right]$ and we have,

$$
B^{T} B=\mathbf{I}
$$

where $\mathbf{I}$ is the identity matrix. Laguerre parameters $L$ and $a$ are suppressed in $B$.

In this way, fIRF is parameterized by the expansion coefficients $c=\left[c_{0}, c_{1}, \ldots, \mathcal{c}_{L-1}\right]^{T}$ on a Laguerre basis set with pre-specified scale and dimension (see section 3.4). Therefore, deconvolution of fIRF $h(t)$ consists of estimating its Laguerre expansion coefficients $\boldsymbol{c}$.

\subsection{Ordinary least-square deconvolution with Laguerre expansion (OLSD-LE)}

Since Laguerre expansion (equation (2)) provides a linear parameterization of fIRF, LSD can be solved using OLS method. Here we briefly summarize the mathematical formulation of OLSD-LE. Under the Laguerre expansion, discrete time measured signal (equation (1)) becomes,

$$
\begin{aligned}
y(k) & =\sum_{i=1}^{k} \sum_{l=0}^{L-1} c_{l} I(k-i) b_{l}(i ; \alpha)+\varepsilon_{k} \\
& =\sum_{l=0}^{L-1} c_{l} v_{l}(k ; \alpha)+\varepsilon_{k}
\end{aligned}
$$

where we defined $v_{l}(k ; \alpha)=\sum_{i=1}^{k} I(k-i) \cdot b_{l}(i ; \alpha)$. 
To simplify notation, we define $\boldsymbol{y}=[y(1), y(2), \ldots, y(N)]^{T}, \boldsymbol{h}=[h(1), h(2), \ldots, h(N)]^{T} \boldsymbol{v}_{l}=$ $[V(1 ; a), V(2 ; a), \ldots, V(N ; a)]^{T}, \mathcal{E}=\left[\mathcal{E}_{1}, \mathcal{E}_{2}, \ldots, \mathcal{E}_{N}\right]^{T}$ such that $(6)$ can be written in a matrixvector form,

$$
\boldsymbol{y}=V c+\varepsilon
$$

Here, we suppressed the dependence of $V$ on basis parameters $L$ and $a$.

OLSD-LE estimates the Laguerre expansion coefficients $c$ by solving an OLS problem,

$$
\underset{c \in R^{L}}{\operatorname{minimize}}\|\boldsymbol{y}-V c\|^{2}
$$

which has solution of $\widehat{c}_{\mathrm{OLS}}=\left(V^{T} V\right)^{-1} V^{T} \boldsymbol{y}$. Note that direct matrix inversion is not recommended in most practical applications. In addition, the OLS estimate of fIRF $\boldsymbol{h}$ is given by

$$
\widehat{\boldsymbol{h}}^{(\mathrm{OLS})}=B \widehat{c}_{\mathrm{OLS}}
$$

Unlike multi-exponential expansion, expansion of fIRFs with LBFs is more justified from its mathematical simplicity and numerical fitness other than from its description of relaxation dynamics of physical fluorescing systems. The fIRFs from physical systems are believed to be "decay" functions that smoothly decay to zero at long enough time delays. However, the inherent oscillatory nature of LBFs can give rise to unphysical behavior of fluorescence decay functions using OLSD-LE, for measurements with relatively low SNRs (e.g. SNR < 30dB), especially when higher-order LBFs are used in expansion.

\subsection{Constrained least square deconvolution with Laguerre expansion (CLSD-LE)}

We introduce here CLSD-LE as a means of addressing the limitations of OLSD-LE when used for the deconvolution of fIRF. OLSD-LE deconvolution searches for the fIRF embedded in a linear space spanned by the set of LBFs, which has minimal distance to timeresolved measurement. The function found, however, does not necessarily agree with the $a$ priori knowledge of the shape of decay functions of fluorescent physical systems. Thus, we considered "constraining" the search within the subspace of functions that are physically realistic and therefore candidates of "decay" functions. To make this argument more rigorous, in this study, the following definition was used.

Definition. A real analytic function $h(t)$ for $0 \leq t<\infty$ is a candidate fIRF, if (i) $\lim _{t \rightarrow \infty} h(t)=0$, (ii) $h^{\prime}(t)<0$, (iii) $h^{\prime \prime}(t)>0$. where $h^{\prime}(t)$ and $h^{\prime \prime}(t)$ are first and second derivatives of $h(t)$. Note that conditions (i) and (ii) guarantee $h(t)$ to be positive. Therefore the fIRF is necessarily positive, monotonic decrease, strictly convex and asymptotically goes to zero. It should also be noted that we required the second derivative of $h(t)$ to be strictly positive so that there are no "flat" (i.e. zero curvature) line segments on a decay profile. Although, this definition does not capture all features of a smooth "decay" function, such conditions allow us to limit our search of fIRF within a smaller subspace of functions that are more physically realistic and are strong enough for practical uses as demonstrated in this study.

Strict inequality constraints as in the above definition is difficult to handle in most CLS solvers, so we use the following simplified condition. 
Lemma. Let $h^{\prime \prime \prime}(t)$ be the third derivative of $h(t)$. For $t \geq 0$, if (a) $\lim _{t \rightarrow \infty} h(t)=0, \lim _{t \rightarrow \infty} h^{\prime}(t)=0, \lim _{t \rightarrow \infty} h^{\prime \prime}(t)=0$, and (b) $h^{\prime \prime \prime}(t) \leq 0, h(t)$ is a candidate fIRF.

This is because, given $h^{\prime \prime \prime}(t) \leq 0$ for $t \geq 0, h^{\prime \prime}(t)$ is a monotonic nonincreasing function. Then because $\lim _{t \rightarrow \infty} h^{\prime \prime}(t)=0$, there must exist a $t^{*} \geq 0$ such that $h^{\prime \prime}(t)>0$ for $t<t^{*}$ and $h^{\prime \prime}(t)=$ $0 t \geq t^{*}$. But for the real analytic function $h(t), t^{*}$ must be infinity, because if not, $h(t)$ has to be linear in $t$, i.e. $h(t)=h\left(t^{*}\right)+h^{\prime}\left(t^{*}\right)\left(t-t^{*}\right)$, which gives $h^{\prime \prime}(t)=0$ for $t \geq 0$. This is in contradiction to $h^{\prime \prime}(t)>0$ for $t<t^{*}$. Thus, $h^{\prime \prime}(t)>0$ for $0 \leq t<\infty$. Similarly we can obtain $h$ $'(t)<0$ and $h^{\prime \prime}(t)>0$ for $0 \leq t<\infty$. Therefore, $h(t)$ is strictly convex, positive, and monotonic decreasing for $0 \leq t<\infty$.

Note that for multi-exponential expansion of fIRF, $h(t)$ is analytic. In addition, constraints (a) and (b) in Lemma are automatically satisfied. For $h(t)$ expanded on the set of LBFs (equation (2)), its analyticity and asymptotic behavior are determined by the expansion basis functions. Following the definition of continuous time LBFs (Abramowitz and Stegun, 1973), it is easy to verify that LBFs are analytic functions and all orders of derivatives of LBFs go to zero for $t \rightarrow \infty$. Thus, in principle, condition (a) is also satisfied for Laguerre expansion of $h(t)$. In practice, time-resolved measurements for evaluating the fluorescence decays are always truncated to a finite range. It is important to choose an appropriate Laguerre basis set such that all its basis functions and corresponding derivatives decay "sufficiently close" to zero at the end of the measured time series. In other words, constraint (a) is imposed implicitly in choosing the expansion basis set (see section 3.4.1).

Then, $h(t)$ further subject to constraint (b) can be found by solving the following CLS problem for Laguerre expansion coefficient $c$,

$$
\begin{array}{ll}
\underset{c \in R^{L}}{\operatorname{minimize}} & \|\boldsymbol{y}-V c\|^{2} \\
\text { subject to } & D^{(3)} B c^{\circ} \quad 0
\end{array}
$$

where $D^{(3)}$ is the third order forward finite difference matrix (Abramowitz and Stegun, $1973)$ of size $(N-3) \times N$. Note that in discrete time representation, $h\left(t_{k}\right)=\sum_{l=0}^{L-1} B_{k l} c_{l}$. The constraint in CLS problem (10) is the discrete-version of the non-positivity condition for the third derivative of fIRF, i.e. constraint (b) in Lemma.

Introducing Lagrangian multiplier $\lambda$ and defining $D=D^{(3)} B$ and defining results in the Lagrangian dual problem (Boyd and Vandenberghe, 2004) to (10),

$$
\begin{array}{cc}
\underset{\lambda \in R^{N-3}}{\operatorname{minimize}} & \left\|C\left(V^{T} \boldsymbol{y}-D^{T} \lambda\right)\right\|^{2} \\
\text { subject to } & \lambda \pm 0
\end{array}
$$

where $C$ is the Cholesky decomposition of positive definite matrix $\left(V^{T} V\right)^{-1}$. This nonnegative least square (NNLS) problem is easily solved using the Active Set Method (Lawson and Hanson, 1974). The CLS estimate of fIRF is,

$$
\widehat{\boldsymbol{h}}^{(\mathrm{CLS})}=B \widehat{c}_{\mathrm{CLS}}
$$

where $\widehat{c}_{\mathrm{CLS}}=\left(V^{T} V\right)^{-1}\left(V^{T} \boldsymbol{y}-D^{T} \widehat{\lambda}\right)$ and $\widehat{\lambda}$ is the solution to NNLS problem (11). 


\subsection{Average lifetimes}

In the context of TRFS and FLIM, of particular interest is the average lifetime known as the conventional parameter capturing the characteristics of the fluorescence decay. This can be estimated from the deconvolved decay function $\hat{h}(k)$ as (Lakowicz, 2006),

$$
\widehat{\tau}_{\mathrm{avg}}=\frac{\delta t \sum_{k=1}^{N} \widehat{k h}(k)}{\sum_{k=1}^{N} \widehat{h}(k)}
$$

Note that definition in equation (13) is consistent with conventional definition of lifetimes when time-resolved fluorescence signals are analyzed using multi-exponential expansion. For a one-component fluorescence system, whose decay function is a single exponential function, this is the time required for the fluorescence intensity to decrease to $1 / e$ of its maximum value. In the presence of multi-exponential decay components, the average lifetime defined in equation (13) is the mean of lifetimes from individual exponential components weighted by their fractional contributions (see section 3.1.1). However, the estimation of average lifetimes using equation (13) does not require the knowledge of lifetimes from individual species and their corresponding fractional contributions.

\section{Methods}

\subsection{Simulated data and validation methods}

3.1.1 Generation of simulated data-A series of computer simulated data were used for the quantitative analysis of CLSD-LE method. We simulated fluorescence systems consisting of exponential decay components (single or multiple). Time-resolved measurements were generated according to the convolution of simulated multi-exponential fIRF and a pre-measured iIRF.

$$
y\left(t_{k}\right)=\sum_{i=1}^{k} I\left(t_{k}-t_{i}\right)\left\{\sum_{j=1}^{M} A_{j} \exp \left[-\frac{t_{i}}{\tau_{j}}\right]\right\}+\varepsilon_{k}
$$

for $k=1, \ldots, 800$ (i.e. 800 sampling time points with sampling interval $0.05 \mathrm{~ns}$ ), where and $A_{j}$ and $\tau_{j}$ are the amplitude and lifetime for the $f^{\text {th }}$ exponential component respectively, and $M$ is the total number of exponential components. The fractional contribution for $J^{\text {th }}$ component is given by (Lakowicz, 2006)

$$
f_{j}=\frac{A_{j} \tau_{j}}{\sum_{k=1}^{M} A_{k} \tau_{k}} \times 100 \%
$$

for $j=1, \ldots, M$ and $\sum_{j=1}^{M} f_{j}=100 \%$. The average lifetime for multi-exponential IRF can be computed from

$$
\tau_{\mathrm{avg}}=\sum_{j=1}^{M} f_{j} \tau_{j}
$$

For a given set of fractional contributions and lifetimes of exponential components, it is possible to compute the ratio of amplitudes $\left(A_{j}\right)$ between any two components. Since our main interest is the estimation of average lifetime, which is not related to absolute fluorescence intensities, the synthetic time-resolved measurements were normalized such that the peak intensity has the value of one. 


\subsubsection{Comparison of CLSD-LE versus other deconvolution techniques-The}

advantage of using the CLSDLE method is demonstrated here by comparing its performance with the OLSD-LE and the conventional LSD with bi-exponential expansion of fIRFs (LSDBE) (Lakowicz, 2006). In this comparison, random samples were generated with additive white Gaussian noise level ranging from $20 \mathrm{~dB}$ to $50 \mathrm{~dB}$. At each noise level, a set of 1000 time-resolved signals was generated according to equation (14). For each signal, the number of exponential components $M$ was randomly picked from 1 to 6 . For each component, a random fractional contribution (uniformly distributed between $0 \%$ and $100 \%$ ) and a random lifetime value (uniformly distributed between $1 \mathrm{~ns}$ and $6 \mathrm{~ns}$ ) were assigned.

Subsequently, the simulated data were deconvolved using CLSD-LE, OLSD-LE and LSDBE methods. The average lifetime of the deconvolved fIRFs were computed using and compared against the ground truth calculated from equation (16). The bias and variance properties of average lifetime estimates from these three methods were compared.

3.1.3 Comparison of CLSD-LE with different Laguerre basis sets-Most of the previous studies using OLSD-LE relied on lower-dimensional ( $L \leq 5$ ) Laguerre expansions of fIRF to guard against "overfitting" to noisy measurements. The purpose of this comparison is to show that for systems with multiple fluorescence components, by employing CLS, higher-order LBFs could be used for deconvolving fIRF without "overfitting". In addition, we show that, the CLSD-LE is capable of recovering a wide range of fIRFs with a fixed (and non-optimal) scale parameter and a basis dimension.

We considered fIRF consisting of one fast-decay component (with lifetime $\tau_{1}$ and fractional contribution $f_{1}$ ) and one slow-decay component (with lifetime $\tau_{2}$ and fractional contribution $f_{2}=1-f_{1}$ ). Time-resolved data according to equation (14) were simulated for $f_{1}$ from $0 \%$ to $100 \%$ ( $10 \%$ interval). For each $f_{1}, 1000$ realizations of $25 \mathrm{~dB}$ additive noise were also generated to quantify the bias and variance of estimated average lifetimes. To demonstrate the limit of different Laguerre basis sets, two combinations of $\tau_{1}$ and $\tau_{2}$; were used for the above simulations, i.e. (1) $\tau_{1}=0.5 \mathrm{~ns}$ and $\tau_{2}=6 \mathrm{~ns}$ (2) $\tau_{1}=1 \mathrm{~ns}$ and $\tau_{2}=6 \mathrm{~ns}$.

CLSD-LE with basis dimension $L \in\{4,8,12\}$ were used to deconvolve simulated timeresolved data. For Laguerre basis set with $L=4, a$ was adaptively selected along with the expansion coefficients using optimization (see section 3.4.3). For $L=8$ and $L=12, a$ values were fixed at 0.96 and 0.94 respectively (see section 3.4). CLSD-LE with these three Laguerre basis set are denoted as CLSD-LE(4,optimal), CLSD-LE(8,0.96) and CLSD$\mathrm{LE}(12,0.94)$ respectively.

\subsection{Experimental data and validation methods}

The proposed CLSD-LE method was also validated on experimental TRFS and FLIM data from standard fluorescent dyes, tissue biomolecules, and biological tissues measured in vivo and ex vivo.

3.2.1 Instrumentation-Two instrumental systems were used for the acquisition of experimental data used for the validation of the deconvolution method presented in this study. These systems were described in detail elsewhere (Sun et al., 2009a; Sun et al., 2011b). A brief description of these systems is given in the following:

TRFS Instrumentation: The system consisted of a pulsed nitrogen laser $(337 \mathrm{~nm}, 800 \mathrm{ps}$ pulse width, $2 \mu \mathrm{J} /$ pulse excitation energy, $30 \mathrm{~Hz}$ repetition rate) excitation source, and a gated MCP-PMT (180 ps rise time, $1.5 \mathrm{GHz}$ bandwidth) detector. Sample fluorescence was excited and collected using a single fiber optic. Sample fluorescence signals were spectrally resolved using a monochromator before reaching the detector. The PMT signals were 
recorded and temporally resolved using a digital oscilloscope ( $20 \mathrm{GHz}$ sampling rate, 2.5 $\mathrm{GHz}$ bandwidth). Time-resolved fluorescence response pulses were recorded within 360-600 $\mathrm{nm}$ wavelength range (5 $\mathrm{nm}$ spectral resolution).

FLIM instrumentation: This consisted in a novel scanning FLIM system that shared the same laser excitation source, detector and digitizer as the TRFS system. However, only discrete spectral bands of time-resolved fluorescence data $(390 \pm 20 \mathrm{~nm}, 450 \pm 23 \mathrm{~nm}, 540 \pm 23$ $\mathrm{nm}$, and $630 \pm 26 \mathrm{~nm}$ ) were measured by using dedicated filters for faster acquisition speeds (few microseconds) at one spatial point measurement. Sample scanning was achieved via a precision positioning stage that enabled linear scanning ( $\mathrm{x}$ - resolution $0.081 \mathrm{~mm}$ and $\mathrm{y}$ resolution $0.2 \mathrm{~mm}$ ) for dynamic acquisition of fluorescence data.

3.2.2 Fluorescent dyes and biomolecules-TRFS measurements were conducted in a mixture of two different fluorophores in solution: $2 \mathrm{mM}$ reduced nicotinamide adenine dinucleotide (NADH, Sigma-Aldrich) in phosphate buffered saline (PBS, Sigma-Aldrich) solution was mixed with $1 \mathrm{mM}$ fluorescein (Sigma-Aldrich) PBS solution. The two fluorophores are characterized by fluorescence emission peak of $460 \mathrm{~nm}$ and $520 \mathrm{~nm}$, respectively, and distinct lifetimes (Dabir et al., 2009; Arık et al., 2005). The solution was measured in a polymethyl methacrylate (PMMA) UV transparent cuvette. Data were collected for the 480-600 nm wavelength range.

FLIM measurements: were conducted in spatially distributed capillary tubes filled with four fluorophores: NADH (2 mM, in PBS), coumarin 1 (C-1, Sigma-Aldrich, $1 \mathrm{mM}$, in methanol), coumarin 120 (C-120, Sigma-Aldrich, 1mM, in methanol), and elastin (dry powder form, from bovine neck ligament (E1625-5G), Sigma-Aldrich). The fluorophores were sealed in separate capillary tubes (negligible fluorescence, $0.75 \mathrm{~mm}$ inner diameter) and placed along y-direction approximately $2 \mathrm{~mm}$ apart in $\mathrm{x}$-direction (center-to-center) with an increasing lifetime sequence. Time domain signals were dynamically acquired over $9.72 \times 4.1 \mathrm{~mm}^{2}$ area with an $\mathrm{x}$-scan step size $0.081 \mathrm{~mm}$ and $y$-scan step size $0.1 \mathrm{~mm}$. The final FLIM images consisted of 120 pixels in $\mathrm{x}$-direction and 41 pixels in $\mathrm{y}$-direction.

3.2.3 Biological tissue in vivo and ex vivo measurements-To validate the CLSDLE method on data from biological tissue samples, we utilized existing TRFS and FLIM data in our laboratory from tissue measurements conducted in support of other studies. In brief, TRFS data were based on measurements conducted in vivo on a hamster buccal pouch carcinogenesis model (Syrian/golden hamsters) as described by Sun et al. (2009b) and Farwell et al (2010). Data were collected from the inverted cheek pouch with the fiberoptic probe positioned perpendicular to the regions of interest (normal or tumor) using the TRFS apparatus described above. Tissue time-resolved emissions were recorded for 360-600 nm wavelength range. FLIM data were based on measurements conducted on ex-vivo human carotid atherosclerotic plaque samples as reported by Sun et al. (2011a).

\subsection{Assessing the quality-of-deconvolution}

3.3.1 Bias and variance of estimating average lifetimes-Simulated data allow for direct comparison of estimated average lifetimes $\widehat{\tau}_{\text {avg }}$ (equation (13)) from noise corrupted data with known "true" average lifetime, $\tau_{\text {avg }}$ (e.g. equation (16)). For a set of $N_{S}$ randomly generated fluorescence decays, the estimation errors in estimating average lifetimes were computed as $\left\{\widehat{e}_{n}=\widehat{\tau}_{\text {avg }}^{(n)}-\tau_{\text {avg }}^{(n)}\right.$, for $\left.n=1, \ldots N_{s}\right\}$. Bias and variance of the average lifetime estimator $\widehat{\tau}_{\text {avg }}$ are therefore the sample mean and variance of the estimation errors, respectively. In general, less bias and variance are preferred. In addition, the mean squared 
error (MSE) were computed as the sample mean of $\left\{\widehat{e}_{n}^{2}\right.$, for $\left.n=1, \ldots N_{s}\right\}$ and were also compared.

3.3.2 Summary statistics of residuals-The convolution of estimated fIRF $\hat{h}(i)$ and instrumental response gives the fitted values of time-resolved signals in time domain, i.e.

$$
\widehat{y}(k)=\sum_{i=1}^{k} I(k-i) \widehat{h}(i)
$$

for $k=1, \ldots, N$. Quantitative assessment of the quality of deconvolution can be based on the analysis of residuals between measured time domain signals and fitted values, i.e. $r(k)=y(k)$ $-\hat{y}(k)$, for all sampling times $t_{k}$. Standardized residual (i.e. residuals normalized to an estimate of its standard deviation) and residual autocorrelations as a function of time lags are often used for diagnosing abnormalities of deconvolving single time domain signal. In practice, we consider a deconvolution "good enough", if the fitting residuals follow closely to the underlying noise model and are absent of autocorrelation structures.

For simultaneously assessing the quality of fit from multi-channel measurements, we used summary statistics for residuals as described below.

$\chi^{2}$ goodness-of-fit: Ideally, the residuals should not contain any unfitted component other than random noise. A direct measure of unfitted component against noise is based on $\chi^{2}$ statistic,

$$
\frac{\chi^{2}}{\mathrm{DOF}}=\frac{1}{(N-L) \sigma^{2}} \sum_{k=1}^{N} r(k)^{2}
$$

where $N$ is the total number of time sampling points, $L$ is the number of fitting parameters (i.e. number of coefficients in Laguerre expansion), and $\sigma^{2}$ is the variance of noise. For ideal fit, $\chi^{2}$ / DOF is close to one, reflecting sample variance of residuals is comparable to noise. The $\chi^{2}$ statistic relies on a good estimate of noise variance, $\sigma^{2}$ and was only used in simulation study where noise variance was given.

Lilliefors test statistic: Because the instrumental random noise is assumed to be drawn from a normal distribution, the Lilliefors test of normality for residuals is used. The Lilliefors test statistic, (similar to the Kolmogorov-Smirnov test statistic), is defined as,

$$
T_{\text {Lillie }}=\sup _{x}\left|F_{\text {res }}(x)-F_{\text {Normal }}(x)\right|
$$

where the empirical distribution function for residuals, $F_{\text {res }}(x)$, is defined as $F_{\text {res }}(x)=\sum_{k=1}^{N} I\left[r\left(t_{k}\right)<x\right] / N$, with $\left.\Pi \cdot\right]$ an indicator function. $F_{\text {Normal }}(x)$ is the cumulative distribution function of normal distribution with mean and variance estimated from the sample of residuals. The Lilliefors test statistic measures the deviance of sampling distributions of residuals from normal distribution. Under the null hypothesis that the fit residuals follow normal distribution, $T_{\text {Lillie }}$ is distributed as the Lilliefors distribution.

Ljung - Box test statistic: The independence of residuals is tested using the Ljung-Box test statistic given by 


$$
T_{\mathrm{LB}}=N(N+2) \sum_{k=1}^{20} \frac{\rho(k)^{2}}{N-k}
$$

where $\rho(k)$ is the sample autocorrelation at time lag $t_{k}$. The Ljung - Box test measures the distance from zero of autocorrelation functions average over a number of time lags. Under the null hypothesis that residuals are independently distributed, $T_{\mathrm{LB}}$ follows $\chi^{2}$ distribution with degree-of-freedom (d.o.f.) 20.

Detailed discussion of the Lilliefors and Ljung-Box summary statistics is out of the scope of this paper. It should be noted that, although formal summary statistics provide an efficient way of detecting abnormalities within deconvolution residuals (i.e. unfitted components other than measurement noise), no real measurement noise strictly follows an independent normal distribution. Non-normality and correlation can either be caused by limited bandwidth of detector frequency responses, or be intrinsic to signal sampling schemes, such as those based on time-correlated single-photon counting (TCSPC) technique. Therefore deconvolution residuals should be appropriately weighted (e.g. in case of Poisson distribution of measurement noise) or down-sampled (e.g. in case that sampling frequency is higher than the maximum detector frequency response) before being tested against the hypotheses of normality and independence.

\subsection{Choosing Laguerre parameters}

In principle, if fIRF is expanded on an infinite number of LBFs $(L \rightarrow \infty)$ over infinite long time intervals, the choice of scale parameter is arbitrary ( $0<a<1)$. In practice, only finite number of expansion terms can be used and the measured time series are always truncated for numerical analysis. Thus, appropriate selection of Laguerre basis set is critical for practical use of Laguerre expansion of fIRFs.

In the following it is always assumed that the time domain fluorescence measurements have been properly truncated prior to any deconvolution process. This means, for a TRFS or FLIM data set (consisting of multiple fluorescence emissions from fIRFs with multiple decay components), a finite time interval should be selected such that measured signal intensity from the overall "slowest" decay component within the data set is truncated not long after it decays to zero.

3.4.1 Upper bound of $\boldsymbol{\alpha}$-To ensure the mutual orthonormality of Laguerre basis set with $L$ LBFs over a finite number of sampling points, it is necessary to choose $a$ values such that $B^{T} B$ is close to identity matrix (equation (5)). This requires that the condition number of matrix $B^{T} B$ being close enough to one (e.g. cond $\left(B^{T} B\right) \leq 1.01$ ), which set an upper bound of $a, a_{\mathrm{up}}$, because cond $\left(B^{T} B\right)$ is a non-decreasing function of $a$. Moreover, the mutual orthonormality within Laguerre basis set also guarantees its basis function and corresponding derivatives reach zero (approximately) by the end of a truncated time interval. Thus, constraint (a) in the Lemma is implicitly imposed by choosing Laguerre basis sets that preserve the orthonormality over the time interval of interests. Table 1 summarizes the upper bounds of $a$ values for two truncated time intervals (20 ns and $40 \mathrm{~ns}$ ) with sampling rate $0.05 \mathrm{~ns}$ and $0.125 \mathrm{~ns}$, for basis sets with 4,8 and $12 \mathrm{LBFs}$.

3.4.2 Practical consideration-For deconvolution of tissue fIRF from TRFS and FLIM measurements, selection of Laguerre parameters of $L$ and $a$ was based on two more practical considerations: first, fluorescence average lifetime values for common tissue endogenous fluorophores often range from sub-nanosecond (lipid constituents) to 
approximately 6 ns (structural proteins such as collagen); second, it is often desired to simultaneously deconvolve multi-channel signals from spectral and spatial scans. Our goal in designing the appropriate basis set for CLSD-LE is to find a basis set with a fixed number of LBFs and scale parameter, that is "sufficiently accurate" for recovering fIRFs with lifetimes ranging from $0.5 \mathrm{~ns}$ to $6 \mathrm{~ns}$.

In this study, Laguerre basis set was selected using the following simulation. The discrete time-resolved signals were simulated from single exponential fIRF according to equation (14) with the lifetime value $\tau$ within the desired range (from $0.5 \mathrm{~ns}$ to $6 \mathrm{~ns}$ ). For each $\tau$ value, 200 realizations fluorescence signals with $25 \mathrm{~dB}$ additive normal noise were generated. All simulated signals were deconvolved using CLSD-LE with Laguerre basis sets of various combinations of $a$ and $L$ values. In this work, we limited $L$ values to be 4,8 and 12. For each $L$, scale parameter $a$ values were chosen from 0.9 to its corresponding upper bound for 800 sampling points (i.e. $a_{\text {up }}=0.98,0.96$ and 0.94 for $L=4,8$ and 12 respectively). Bias and variances in average lifetimes from CLSD-LE with different Laguerre bases were computed for each "true" lifetime. Figure 1a depicts the bias surface and variance surface on the support of "true" lifetime values and $a$ values for $L=8$. This process was repeated for $40 \mathrm{~dB}$ additive noise.

As illustrated in figures $1 \mathrm{~b}, 1 \mathrm{c}$ and $1 \mathrm{~d}$, for each $L$ value, there is a region (e.g. region between thick black lines) on $a-\tau$ plane where squared biases are less than variances in estimating lifetimes and the estimation error could be attributed mainly to random measurement noise. The chosen $a$ and $L$ were considered "sufficiently accurate" for deconvolving fIRFs with lifetimes in this range in practice. Obviously, acceptable region shrinks as SNR increases. In addition, the sampling time interval of our current instrument was around $0.05 \mathrm{~ns}$. Lifetime estimated with biases less than $0.05 \mathrm{~ns}$ were also considered acceptable in practice.

Therefore, CLSD-LE with 4 LBFs and $a=0.96$ was only acceptable for lifetimes between $1.5 \mathrm{~ns}$ and $4 \mathrm{~ns}$. For simultaneously deconvolving fIRF with lifetimes between $1 \mathrm{~ns}$ and $6 \mathrm{~ns}$, CLSD-LE with $8 \mathrm{LBFs}$ and $a=0.96$ were found sufficient. When very fast decay components (e.g. lifetime less than $1 \mathrm{~ns}$ ) are present, 12 LBFs were needed to provide a wider coverage in the acceptable region.

3.4.3 Searching for $\boldsymbol{\alpha}$ using optimization-In previous work (Dankers and Westwick, 2006; Dabir et al., 2009) employing OLSD-LE, it was proposed to automate the search of $a$ as part of the OLS problem (equation (8)) for a fixed $L$. In current study, this idea is generalized for CLSD-LE. In fact, the search of the scale parameter $a$ can be automated if it is treated as a free optimization parameter in CLS problem (equation (10)), in addition to Laguerre expansion coefficients, $c$. Specifically, for given $L$, the optimal $a \in\left(0, a_{\text {up }}\right)$ minimizes the functional,

$$
\begin{aligned}
\Omega(\alpha) & =\left\|\boldsymbol{y}-V(\alpha) \widehat{c}_{\mathrm{CLS}}(\alpha)\right\|^{2} \\
& =\left\|\boldsymbol{y}-V(\alpha)\left(V(\alpha)^{T} V(\alpha)\right)^{-1}\left(V(\alpha)^{T} \boldsymbol{y}-D(\alpha)^{T} \widehat{\lambda}(\alpha)\right)\right\|^{2}
\end{aligned}
$$

where $\widehat{\lambda}(\alpha)$ is the solution to the NNLS problem (equation (11)). Note that we have made the dependence of matrices $V$ and $D$ on $a$ explicit. Minimization of $\Omega(a)$ consisted of a nonlinear optimization problem and is often solved iteratively. In this study, the Golden Section Search Method (Press, 2007) was used to determine the optimal $a$.

For a given fluorescence measurement, Laguerre basis set with optimal $a$ could be considered as the "best" expansion basis (in the least-square sense) of fIRF for given $L$. For 
TRFS and FLIM applications, when near real time deconvolution of measurements from multiple wavelengths/pixels is required, it is impractical to select basis parameters through nonlinear optimization for all individual measurement. Therefore, in this study, searching for LG parameters using optimization only served for comparison purposes.

\section{Results}

\subsection{Validation of CLSD-LE on simulated data and comparative studies}

\subsubsection{Performance of CLSD-LE deconvolution in comparison with other deconvolution techniques-Table 2 shows the comparison of OLSD-LE, CLSD-LE} and LSD-BE performance applied to simulated random data. Laguerre expansion basis with $8 \mathrm{LBFs}$ and $a=0.96$ was used. Note that the underlying fIRF models used in simulation have random number of exponential decay components. The average lifetimes estimated from LSD-BE had the highest bias in the presence of high noise as a result of model misspecification. However, as the noise level decreased, LSD-BE was capable of deconvolving fIRF with more than two components. This demonstrates that it is not always possible to differentiate decay components purely based on the number of terms used in multi-exponential expansion of fIRFs. OLSD-LE was shown to be more sensitive to noise (e.g. higher variance in $\widehat{\tau}_{\text {avg }}$ ) for signals with low SNR. This is an effect of "overfitting" to noise, when higher-order LBFs ( $L=8$ in this case) were used in Laguerre expansion. In addition, "overfitting" could result in unphysical behavior in the deconvolved fIRFs, such as oscillations (bumps) and the presence of negative values (figure 2). On the other hand, CLSD-LE was robust to noise for all simulated SNR levels and had the lowest RMSE for low SNR (e.g. less than $30 \mathrm{~dB}$ ).

CLSD-LE took more computation time than OLSD-LE, but it was still about 100 times faster than the conventional LSD-BE. Moreover, the computation time of CLSD-LE decreased as SNR increased. This is because, in the Active Set Method for solving NNLS in this study (equation (11)), more "active" sets are activated when noise level is high, which results in an increase in computation cost. More importantly, although CLSD-LE was slower than OLSD-LE, it took less than 1 second for deconvolving 1000 simulated measurements. The computations in this study were carried out on a PC laptop (Intel Core i7 Q720 1.60 $\mathrm{GHz})$.

4.1.2 Comparing CLSD-LE using different Laguerre basis sets-Shown in figures $3 \mathrm{a}$ and $3 \mathrm{~b}$ are the $\chi^{2}$ values for CLSD-LE with different Laguerre basis sets in cases of fIRFs with two decay components and varying fractional contributions $\left(\Delta f=f_{1}-f_{2}\right)$. Note that for CLSD-LE with 4 LBFs, $a$ was chosen based on optimization (section 3.4.3). It was observed that even for the optimal $a$, CLSD-LE with 4 LBFs was not able to sufficiently fit the data for most of $\Delta f$ values. In fact, the only situation where CLSD-LE(4,optimal) could fit the generated data adequately was when the sample fluorescence decays become more "homogeneous" (i.e. almost purely from either fast- or slow- decay component, or equivalently $\mid \Delta f \approx \approx 100 \%)$. The performance of CLSD-LE $(8,0.96)$ depended on the presence of the short-decay component (i.e. $\tau_{1}=0.5 \mathrm{~ns}$ and $\tau_{2}=6 \mathrm{~ns}$, CLSD-LE $(8,0.96)$ "failed" to fit the data when the fast-decay component started dominant the decay function (e.g. $\Delta f>$ 0 ). However, in the second case, the lifetime values of fast-decay component were equal to $1 \mathrm{~ns}$, CLSD-LE $(8,096)$ was able to accurately fit the data. For both cases, LCSD$\mathrm{LE}(12,0.94)$ was able to provide good fit as the $\chi^{2}$ values were close to ideal.

Bias, variance and RMSE of average lifetimes estimators $\widehat{\tau}_{\text {avg }}$ estimated using these three basis sets are compared in table 3 (for $\tau_{1}=0.5 \mathrm{~ns}, \tau_{2}=6.0 \mathrm{~ns}$ ) and table 4 (for $\tau_{1}=1 \mathrm{~ns}, \tau_{1}$ $=6.0 \mathrm{~ns}$ ). In addition, bias, variance and RMSE of $\widehat{\tau}_{\text {avg }}$ from the LSD-BE are also provided. 
Since simulated data were generated from a bi-exponential fIRF, LSD-BE was expected to provide theoretically "the best" estimates for $\widehat{\tau}_{\text {avg }}$. In both cases, CLSD-LE(4,optimal) gave the highest bias in estimating averaged lifetimes (i.e. under-estimate the average lifetimes) which in turn gave the highest RMSE, except for almost "homogeneous" fIRFs, as a result of insufficient fit to data. On the other hand, even without the optimally selected $a$, CLSDLE $(12,0.94)$ provided similar bias and variance (and RMSE) levels for $\widehat{\tau}_{\text {avg }}$ to those from LSD-BE. CLSD-LE $(8,0.96)$ was also able to give the similar bias and variance (and RMSE) to CLSD-LE $(12,0.94)$ and LSD-BE, given the fast-decay component's lifetime values were equal to $1 \mathrm{~ns}$. It was also observed that higher model complexity (e.g. Laguerre basis with 12 LBFs) did not introduce higher variance in estimating average lifetimes if CLSD was used.

Therefore, for practical purposes, we found that except for some sparse cases where short lifetimes (e.g. $0.5 \mathrm{~ns}$ ) are present, CLSD-LE(8,0.96) is sufficient for deconvolving fIRFs with multiple decay components, whose lifetime values range from $1 \mathrm{~ns}$ to $6 \mathrm{~ns}$.

\subsection{Validation of CLSD-LE on TRFS experimental data}

\subsubsection{TRFS measurements of fluorescent dye-biomolecule mixtures-TRFS} measurements from the mixture of fluorescein and NADH for 480-600 nm wavelength range were first deconvolved using LSD-BE. Figure 4a shows lifetime spectra of two decay components. The slow-decay component had constant lifetimes, $3.9 \pm 0.1 \mathrm{~ns}$, for 500-600 $\mathrm{nm}$ range which correspond to fluorescein lifetimes. The fast-decay component had constant lifetimes, $0.44 \pm 0.08 \mathrm{~ns}$, for $480-600 \mathrm{~nm}$ range which correspond to NADH lifetimes. The corresponding fractional contributions from both components are given in figure $4 \mathrm{~b}$. For shorter wavelength (e.g. $\leq 490 \mathrm{~nm}$ ), the signals were dominated by NADH fluorescence. As wavelength increased above $490 \mathrm{~nm}$, the fluorescence signals had more contributions from fluorescein rather than NADH. However, fluorescein contribution reached maximum around its emission peak wavelength $(520 \mathrm{~nm})$ and started to decrease with increased wavelength. The average lifetime spectrum from LSD-BE is shown in figure $4 \mathrm{c}$.

The average lifetimes from CLSD-LE(4,optimal), CLSD-LE(8,0.96) and CLSD-LE(12,0.94) are given in figure $4 \mathrm{c}$. As expected, average lifetime estimated from CLSD-LE(12,0.94) nearly overlapped with LSD-BE estimates. Average lifetimes estimated from CLSD$\operatorname{LE}(8,0.96)$ were biased for wavelengths shorter than $490 \mathrm{~nm}$ and became increasingly biased for wavelengths above $540 \mathrm{~nm}$ as NADH (fast-decay component) fractional contribution increased. At last, with the optimal value for each wavelength, CLSDLE(4,optimal) still resulted in significant differences from LSD-BE estimates for the entire wavelength range, except for wavelengths at which fluorescence signals were mostly from one component (e.g. shorter than $490 \mathrm{~nm}$ and around $520 \mathrm{~nm}$ ).

4.2.2 TRFS measurements of oral hamster carcinoma in vivo-Fluorescence signals from $360-500 \mathrm{~nm}$ wavelength range were deconvolved using CLSD-LE $(8,0.96)$. The integrated intensity spectrum and estimated average lifetime are shown in figure $5 \mathrm{a}$.

Lilliefors test statistic (figure 5b) and Ljung-Box test statistic (figure 5c) were computed for deconvolution residuals at each wavelength. Note that critical values have to be corrected (Bonferroni correction) for simultaneous test of normality and independence of deconvolution residuals from multiple wavelengths using Lilliefors test and Ljung-Box test. The critical values on significance level 0.001 were also shown in figure $5 \mathrm{c}$. The fact that most test statistic values from both tests were below the critical value suggests that overall deconvolution is "good enough". Figures $5 \mathrm{~d}$, 5e and $5 \mathrm{f}$ show the deconvolution of representative time-resolved measurements at three wavelengths $380 \mathrm{~nm}, 430 \mathrm{~nm}$ and 480 $\mathrm{nm}$, respectively, along with standardized residual and autocorrelation function. 
As demonstrated by previous studies (Farwell et al., 2010), the fluorescence of cheek pouch under UV excitation primarily originated from collagen in sub-mucosa layer and NADH in cells. This is confirmed by the presence of two emission peaks ( $390 \mathrm{~nm}$ and $460 \mathrm{~nm}$ corresponding to collagen and NADH emissions respectively) in integrated intensity spectrum (figure 5a). Moreover, average lifetime values were consistent with previously reported lifetimes of collagen around its emission peak (i.e. $390 \mathrm{~nm}$ ). As a result of the decreasing contribution from collagen and increasing contribution from NADH, the average lifetime values dropped with increased wavelength.

\subsection{Validation of CLSD-LE on FLIM experimental data}

4.3.1 FLIM measurements of fluorescent dyes and biomolecules-The integrated intensity of the measured fluorescence signals from wavelength band of $450 \pm 23 \mathrm{~nm}$ for the four fluorophores in capillary tubes are depicted in figure $6 \mathrm{a}$. The estimated lifetime maps from LSD-BE, CLSD--LE $(8,0.96)$ and CLSD-LE $(12,0.96)$ are provided in figures 6b, 6c, and $6 \mathrm{~d}$.

The mean and standard deviation of estimated average lifetime values are compared in table 5. All three deconvolution methods provided similar lifetime values for C-1, C-120 and elastin which were also consistent with literature values (Rusalov et al., 2004; Pal et al., 2003; Žukauskas et al., 2008). For free-form NADH which has fast-decay dynamics, the CLSD-LE(12,0.94) provided smaller average lifetime than CLSD-LE $(8,0.96)$ but closer to values estimated based on the multi-exponential approach, i.e. LSD-BE. This is in agreement with the simulations study in previous sections.

4.3.2 FLIM measurements of arterial samples ex vivo-A FLIM scan measurement region $\left(15 \times 4 \mathrm{~mm}^{2}\right)$ on the ex vivo tissue sample is shown in figure $7 \mathrm{a}$. The image consists of 183 pixels in $x$-direction and 20 pixels in y-direction. Fluorescence signals from $450 \pm 23 \mathrm{~nm}$ wavelength band were deconvolved with fixed Laguerre basis set, CLSD-LE( $8,0.96)$. The integrated intensity and average lifetime map are shown in figures $7 \mathrm{~b}$ and $7 \mathrm{c}$ respectively.

The Lilliefors test statistic and Ljung-Box test statistic were computed for deconvolution residuals at each pixel within the range of interest (2253 pixels). The sampling distributions of Lilliefors test statistic and Ljung-Box test statistic are shown in figures $7 \mathrm{~d}$ and $7 \mathrm{e}$ respectively. Their corresponding null distributions (i.e. Lilliefors distribution and $\chi^{2}$ distribution with degree of freedom 20 , respectively) are also shown in these figures. The fact that both test statistics followed their respective null distributions is strong evidence that time-resolved signals across all pixels were adequately fitted. Figures $7 \mathrm{f}, 7 \mathrm{~g}$ and $7 \mathrm{~h}$ show the deconvolution of signals from three representative locations (lipid-rich, collagen-rich and elastin-rich respectively as confirmed by histopathologic analysis) along with the corresponding standardized residual and autocorrelation functions.

\section{Discussion and conclusion}

In this study, we have demonstrated a deconvolution method using high-dimensional Laguerre expansion of fIRF. We showed that the use of a large number of LBFs allows for accurate recovery of tissue fluorescence impulse responses with complex decay dynamics including a broad range of decay components (fast and slow). Moreover, multiple channel measurements on systems with a large number of relaxation dynamics could be deconvolved simultaneously, using the same set of basis functions.

The problem of overfitting due to the increased model complexity has been alleviated by properly incorporating a priori knowledge on the shape of physically realistic fluorescence decay profiles. We implemented a deconvolution algorithm using a constrained least-square 
method to explicitly take advantage of available a priori information. While the fIRF models based on superposition of multi-exponential functions are based on "hard" a priori knowledge of the physics and constituents of the underlying fluorescence systems, the method presented in this paper only requires the fIRF to smoothly decay to zero with time. It can be considered as a "soft" a priori assumption on the functional form of IRFs. Specific to this work, a non-positive third-order derivative of fluorescence decay was used as constraint to LSD. The method can be extended to constrain higher order derivatives of decay functions when necessary. The a priori knowledge on the dynamics of underlying fluorescence systems (i.e. the range of lifetime values) was also used for designing Laguerre basis sets such that the selected scale and dimension parameters could provide an adequate representation of the fIRF. The statistical analysis framework presented in this study can also be generalized to choose Laguerre basis parameters for deconvolving fluorescence decays from other biological systems with dynamics out of the range of current work.

The proposed method is also orders of magnitude faster than the conventional deconvolution based on multi-exponential IRF models. The ability of deconvolving multiple spectral and spatial channel (e.g. wavelengths, locations) measurements within several seconds on a common personal computer makes it ideal for real-time robust analysis of fluorescence decays, which supports the advancement of fluorescence lifetime instrumentation for clinical applications.

By testing the sampling distribution of deconvolution residuals against a null normal distribution, it is also observed that the noise structure of our current instrument follows closely a normal distribution. This has not been addressed before. However, a more detailed study of these noise structures is out of the scope of this paper and is subject to future analysis.

In summary, we have developed a fast and robust method to estimate the impulse response function from time-domain fluorescence measurements of biological tissues. This method has been extensively validated on fluorescence data from artificial simulation, dye phantom and in vivolex vivo tissue samples. This approach facilitates analysis of data resulting from time -resolved measurements conducted in tissue in vivo in both spectral and spatial dimensions. Current results support the feasibility of this method for real-time robust analysis of fluorescence decay data and future development of clinical systems with realtime diagnostic capabilities.

\section{Acknowledgments}

This work was supported by the National Institutes of Health (R01 HL 67377 and R21 RR 025818).

\section{References}

Abramowitz, M.; Stegun, IA. Handbook of mathematical functions : with formulas, graphs and mathematical tables. Dover Publications; New York: 1973.

Agrawal A, Gallas BD, Parker C, Agrawal KM, Pfefer TJ. Sensitivity of Time-Resolved Fluorescence Analysis Methods for Disease Detection. IEEE J. Sel. Top. Quantum Electron. 2010; 16:877-85.

Andre JC, Vincent LM, Oconnor D, Ware WR. Applications of Fast Fourier-Transform to Deconvolution in Single Photon-Counting. J. Phys. Chem. 1979; 83:2285-94.

Arık M, Çelebi N, Onganer Y. Fluorescence quenching of fluorescein with molecular oxygen in solution. Journal of Photochemistry and Photobiology A: Chemistry. 2005; 170:105-11.

Boukis C, Mandic DP, Constantinides AG, Polymenakos LC. A novel algorithm for the adaptation of the pole of Laguerre filters. IEEE Signal Process. Lett. 2006; 13:429-32.

Boyd, SP.; Vandenberghe, L. Convex optimization. Cambridge University Press; Cambridge, UK ; New York: 2004. 
Cubeddu R, Comelli D, D'Andrea C, Taroni P, Valentini G. Time-resolved fluorescence imaging in biology and medicine. J. Phys. D: Appl. Phys. 2002; 35:R61.

Dabir AS, Trivedi CA, Ryu Y, Pande P, Jo JA. Fully automated deconvolution method for online analysis of time-resolved fluorescence spectroscopy data based on an iterative Laguerre expansion technique. J. Biomed. Opt. 2009; 14:024030. [PubMed: 19405759]

Dankers, A.; Westwick, D. Nonlinear system identification using optimally selected Laguerre filter banks.; American Control Conference (ACC), 2006. 2006. p. 2369-74.

Dankers, A.; Westwick, DT. A convex method for selecting optimal Laguerre filter banks in system modelling and identification.; American Control Conference (ACC), 2010. 2010. p. 2694-9.

Elson, D.; Galletly, N.; Talbot, C.; Requejo-Isidro, J.; McGinty, J.; Dunsby, C.; Lanigan, P.; Munro, I.; Benninger, R.; Beule, P.; Auksorius, E.; Hegyi, L.; Sandison, A.; Wallace, A.; Soutter, P.; Neil, M.; Lever, J.; Stamp, G.; French, P. Geddes, C.; Lakowicz, J., editors. Springer; US: 2006. p. 477-524.

Farwell DG, Meier JD, Park J, Sun Y, Coffman H, Poirier B, Phipps J, Tinling S, Enepekides DJ, Marcu L. Time-Resolved Fluorescence Spectroscopy as a Diagnostic Technique of Oral Carcinoma Validation in the Hamster Buccal Pouch Model. Arch. Otolaryngol. Head Neck Surg. 2010; 136:126-33. [PubMed: 20157056]

Fu Y, Dumont GA. An Optimum Time-Scale for Discrete Laguerre Network. IEEE Trans. Automat. Contr. 1993; 38:934-8.

Gafni A, Modlin RL, Brand L. Analysis of Fluorescence Decay Curves by Means of Laplace Transformation. Biophys. J. 1975; 15:263-80. [PubMed: 1122338]

Galletly N, McGinty J, Dunsby C, Teixeira F, Requejo Isidro J, Munro I, Elson D, Neil M, Chu A, French P. Fluorescence lifetime imaging distinguishes basal cell carcinoma from surrounding uninvolved skin. Br. J. Dermatol. 2008; 159:152-61. [PubMed: 18460029]

Heuberger, PSC.; Hof, P M J v d; Wahlberg, B. Modelling and identification with rational orthogonal basis functions. Springer; London: 2005.

Isenberg I, Mullooly JP, Dyson RD, Hanson R. Studies on Analysis of Fluorescence Decay Data by Method of Moments. Biophys. J. 1973; 13:1090-115. [PubMed: 4748377]

Jo JA, Fang Q, Papaioannou T, Baker JD, Dorafshar AH, Reil T, Qiao JH, Fishbein MC, Freischlag JA, Marcu L. Laguerre-based method for analysis of time-resolved fluorescence data: application to in-vivo characterization and diagnosis of atherosclerotic lesions. J. Biomed. Opt. 2006; 11:021004. [PubMed: 16674179]

Jo JA, Fang QY, Papaioannou T, Marcu L. Fast model-free deconvolution of fluorescence decay for analysis of biological systems. J. Biomed. Opt. 2004; 9:743-52. [PubMed: 15250761]

König K. Clinical multiphoton tomography. J. Biophotonics. 2008; 1:13-23. [PubMed: 19343631]

Lakowicz, JR. Principles of fluorescence spectroscopy. Springer; New York: 2006.

Lawson, CL.; Hanson, RJ. Solving least squares problems. Prentice-Hall; Englewood Cliffs, N.J.: 1974.

Lee KCB, Siegel J, Webb SED, Leveque-Fort S, Cole MJ, Jones R, Dowling K, Lever MJ, French PMW. Application of the stretched exponential function to fluorescence lifetime imaging. Biophys. J. 2001; 81:1265-74. [PubMed: 11509343]

Lee O, Dumont GA, Tournier P, Wade AP. Analysis of Flow-Injection Peaks with Orthogonal Polynomials. Anal. Chem. 1994; 66:971-82.

Maarek JMI, Marcu L, Snyder WJ, Grundfest WS. Time-resolved fluorescence spectra of arterial fluorescent compounds: Reconstruction with the laguerre expansion technique. Photochem. Photobiol. 2000; 71:178-87. [PubMed: 10687392]

Marcu L, Jo JA, Fang Q, Papaioannou T, Reil T, Qiao JH, Baker JD, Freischlag JA, Fishbein MC. Detection of rupture-prone atherosclerotic plaques by time-resolved laser-induced fluorescence spectroscopy. Atherosclerosis. 2009; 204:156-64. [PubMed: 18926540]

Marmarelis VZ. Identification of Nonlinear Biological-Systems Using Laguerre Expansions of Kernels. Ann. Biomed. Eng. 1993; 21:573-89. [PubMed: 8116911]

McCombie DB, Reisner AT, Asada HH. Laguerre-model blind system identification: cardiovascular dynamics estimated from multiple peripheral circulatory signals. IEEE Trans. Biomed. Eng. 2005; 52:1889-901. [PubMed: 16285393] 
McGinty J, Galletly NP, Dunsby C, Munro I, Elson DS, Requejo-Isidro J, Cohen P, Ahmad R, Forsyth A, Thillainayagam AV. Wide-field fluorescence lifetime imaging of cancer. Biomed. Opt. Express. 2010; 1:627-40. [PubMed: 21258496]

McKinnon AE, Szabo AG, Miller DR. Deconvolution of Photoluminescence Data. J. Phys. Chem. 1977; 81:1564-70.

Murata S, Matsuzaki SY, Tachiya M. Transient Effect in Fluorescence Quenching by ElectronTransfer .2. Determination of the Rate Parameters Involved in the Marcus Equation. J. Phys. Chem. 1995; 99:5354-8.

$\mathrm{Ng}$ BK, Fu CY, Razul SG. Fluorescence lifetime discrimination using expectation-maximization algorithm with joint deconvolution. J. Biomed. Opt. 2009; 14

Niesner R, Peker B, Schlusche P, Gericke KH. Noniterative biexponential fluorescence lifetime imaging in the investigation of cellular metabolism by means of $\mathrm{NAD}(\mathrm{P}) \mathrm{H}$ autofluorescence. ChemPhysChem. 2004; 5:1141-9. [PubMed: 15446736]

O'Connor DV, Ware WR, Andre JC. Deconvolution of Fluorescence Decay Curves - Critical Comparison of Techniques. J. Phys. Chem. 1979; 83:1333-43.

Pal H, Nad S, Kumbhakar M. Photophysical properties of coumarin-120: Unusual behavior in nonpolar solvents. J. Chem. Phys. 2003; 119:443-52.

Pande P, Jo JA. Automated analysis of fluorescence lifetime imaging microscopy (FLIM) data based on the Laguerre deconvolution method. IEEE Trans. Biomed. Eng. 2011; 58:172-81. [PubMed: 20934946]

Press, WH. Numerical recipes : the art of scientific computing. Cambridge University Press; Cambridge: 2007.

Rusalov M, Druzhinin S, Uzhinov B. Intramolecular Fluorescence Quenching of Crowned 7Aminocoumarins as Potential Fluorescent Chemosensors. J. Fluoresc. 2004; 14:193-202. [PubMed: 15615045]

Sun Y, Chaudhari AJ, Lam M, Xie H, Yankelevich DR, Phipps J, Liu J, Fishbein MC, Cannata JM, Shung KK, Marcu L. Multimodal characterization of compositional, structural and functional features of human atherosclerotic plaques. Biomed. Opt. Express. 2011a; 2:2288-98. [PubMed: 21833365]

Sun Y, Park J, Stephens DN, Jo JA, Sun L, Cannata JM, Saroufeem RMG, Shung KK, Marcu L. Development of a dual-modal tissue diagnostic system combining time-resolved fluorescence spectroscopy and ultrasonic backscatter microscopy. Rev. Sci. Instrum. 2009a; 80

Sun YH, Phipps J, Elson DS, Stoy H, Tinling S, Meier J, Poirier B, Chuang FS, Farwell DG, Marcu L. Fluorescence lifetime imaging microscopy: in vivo application to diagnosis of oral carcinoma. Opt. Lett. 2009b; 34:2081-3. [PubMed: 19572006]

Sun YH, Sun Y, Stephens D, Xie HT, Phipps J, Saroufeem R, Southard J, Elson DS, Marcu L. Dynamic tissue analysis using time- and wavelength-resolved fluorescence spectroscopy for atherosclerosis diagnosis. Opt. Express. 2011b; 19:3890-901. [PubMed: 21369214]

Tanguy N, Vilbe P, Calvez LC. Optimum Choice of Free Parameter in Orthonormal Approximations. IEEE Trans. Automat. Contr. 1995; 40:1811-3.

Uehlinger P, Gabrecht T, Glanzmann T, Ballini JP, Radu A, Andrejevic S, Monnier P, Wagnières G. In vivo time-resolved spectroscopy of the human bronchial early cancer autofluorescence. J. Biomed. Opt. 2009; 14:024011. [PubMed: 19405741]

Valeur B. Analysis of Time-Dependent Fluorescence Experiments by Method of Modulating Functions with Special Attention to Pulse Fluorometry. Chem. Phys. 1978; 30:85-93.

Ware WR, Doemeny LJ, Nemzek TL. Deconvolution of Fluorescence and Phosphorescence Decay Curves - Least-Squares Method. J. Phys. Chem. 1973; 77:2038-48.

Westwick, DT.; Kearney, RE. Identification of nonlinear physiological systems. IEEE Press; WileyInterscience; Piscataway, NJ: Hoboken, NJ: 2003.

Yuan HC, Westwick DT, Ingenito EP, Lutchen KR, Suki B. Parametric and nonparametric nonlinear system identification of lung tissue strip mechanics. Ann Biomed Eng. 1999; 27:548-62. [PubMed: 10468239]

Zhang ZY, Grattan KTV, Hu YL, Palmer AW, Meggitt BT. Prony's method for exponential lifetime estimations in fluorescence-based thermometers. Rev. Sci. Instrum. 1996; 67:2590-4. 
Žukauskas A, Vitta P, Kurilčik N, Juršènas S, Bakienė E. Characterization of biological materials by frequency-domain fluorescence lifetime measurements using ultraviolet light-emitting diodes. Optical Materials. 2008; 30:800-5. 

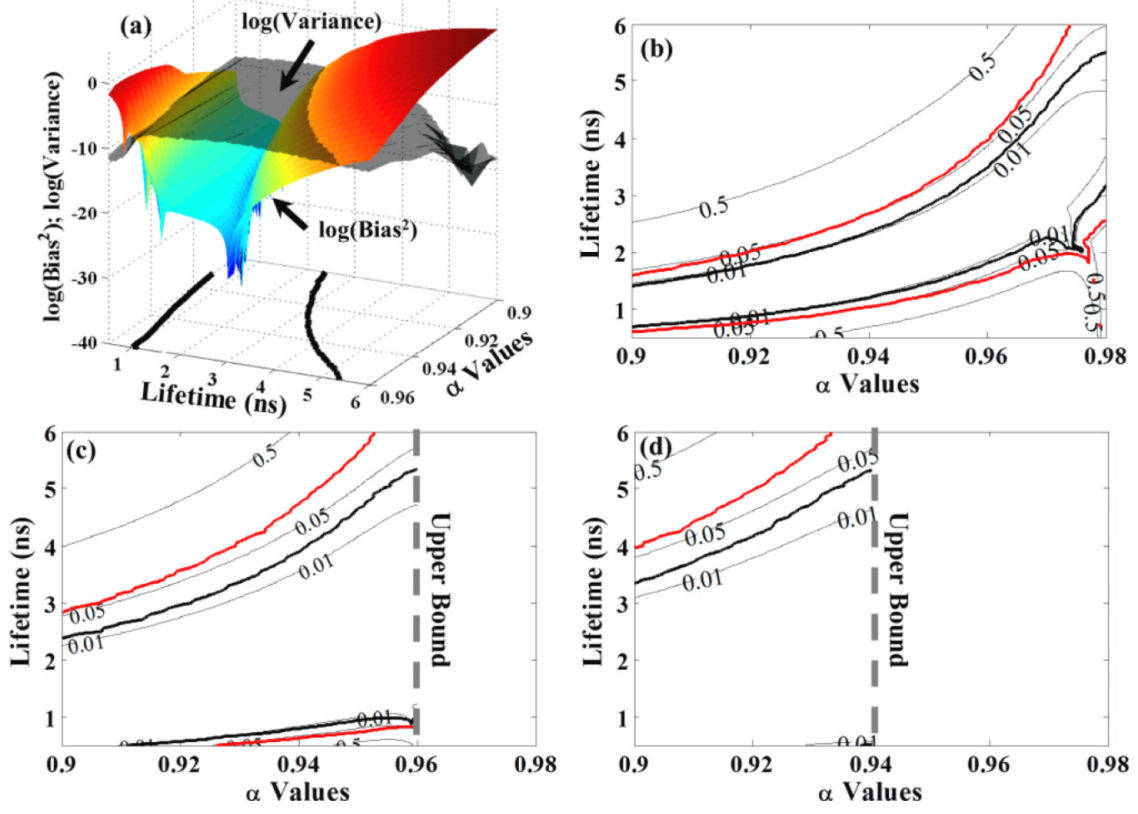

Figure 1.

Analysis of bias and variance in estimating average lifetimes for simulated data from fIRF with true exponential lifetime values between $0.5 \mathrm{~ns}$ and $6 \mathrm{~ns}$. (a) representative bias surface and variance surface from CLSD-LE with Laguerre parameters $L=8, a \in(0.9,0.96)$ for simulated data with additive noise $40 \mathrm{~dB}$; (b) (c) (d) contours (thin black) of bias surface with levels correspond to estimation bias (in absolute value) of $0.01 \mathrm{~ns}, 0.05 \mathrm{~ns}$ and $0.5 \mathrm{~ns}$; intersections between variance surface and bias surface for additive noise $25 \mathrm{~dB}$ (thick red) and $40 \mathrm{~dB}$ (thick black) for CLSD-LE with 4, 8 and 12 LBFs respectively. 


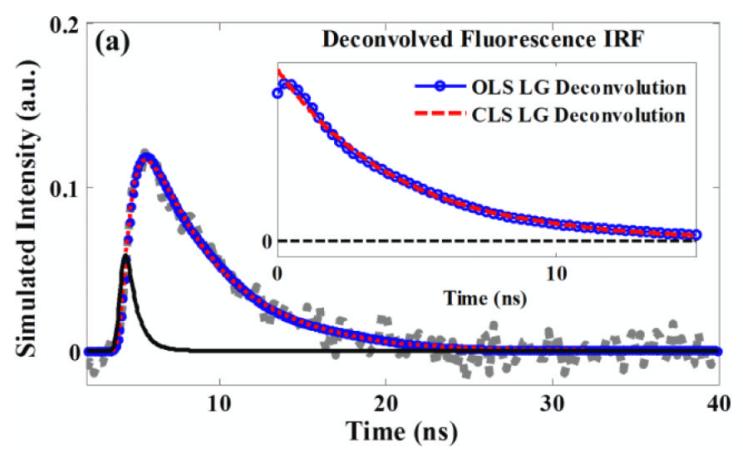

" " - Measured Fluorescence - Instrument IRF

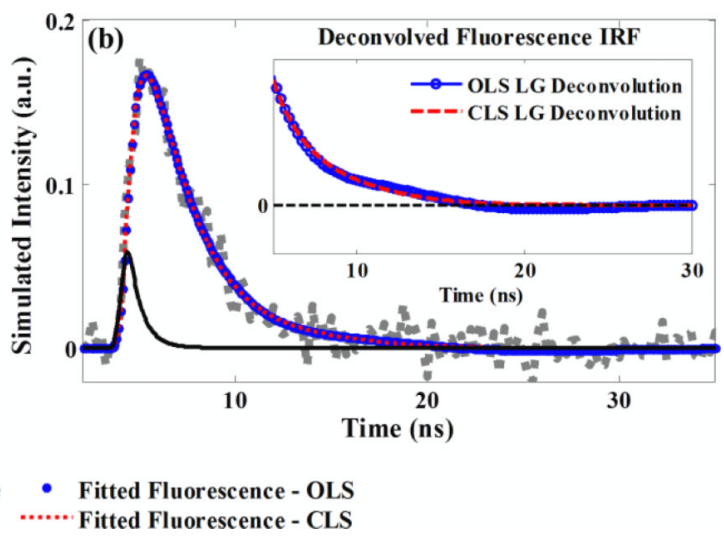

Figure 2.

OLSD-LE and CLSD-LE with Laguerre parameters $L=8$ and $a=0.96$ for two representative signals (a) and (b) (noise level 25dB). Deconvolved fIRF are shown as insets. 

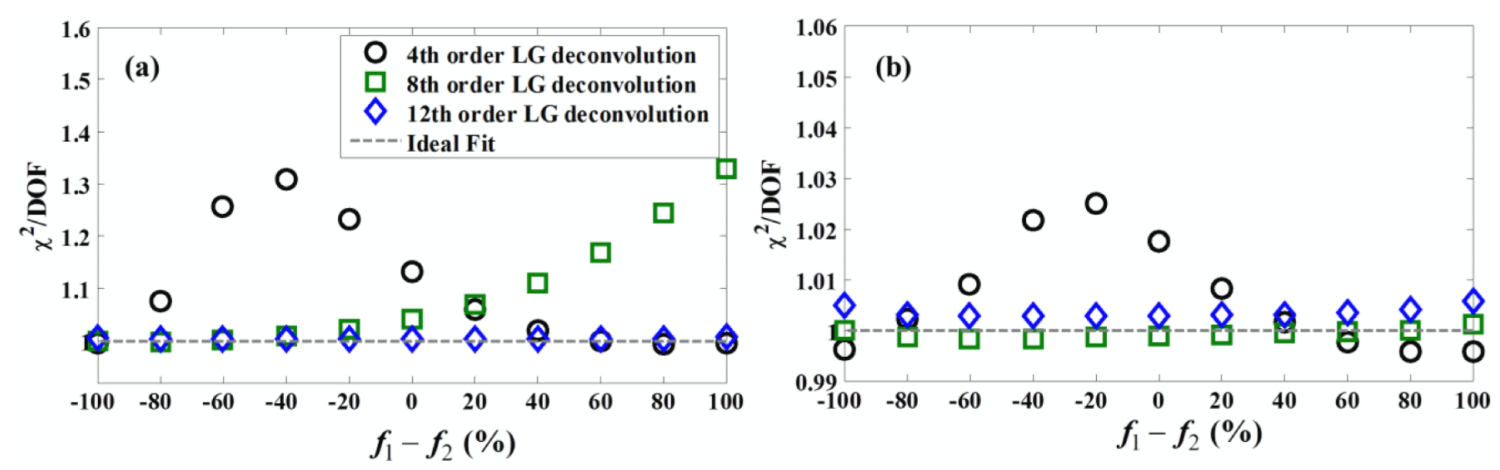

Figure 3.

$\chi^{2}$ goodness-of-fit of CLSD-LE(4,optimal), CLSD-LE(8,0.96) and CLSD-LE(12,0.94) for simulated data from two components fIRFs with different fractional contributions.

Fractional contribution difference $\Delta f$ varies from $-100 \%$ to $100 \%$. (a) $\tau_{1}=0.5 \mathrm{~ns}$ and $\tau_{2}=6$ $\mathrm{ns}$ and (b) $\tau_{1}=1 \mathrm{~ns}$ and $\tau_{2}=6 \mathrm{~ns}$. 

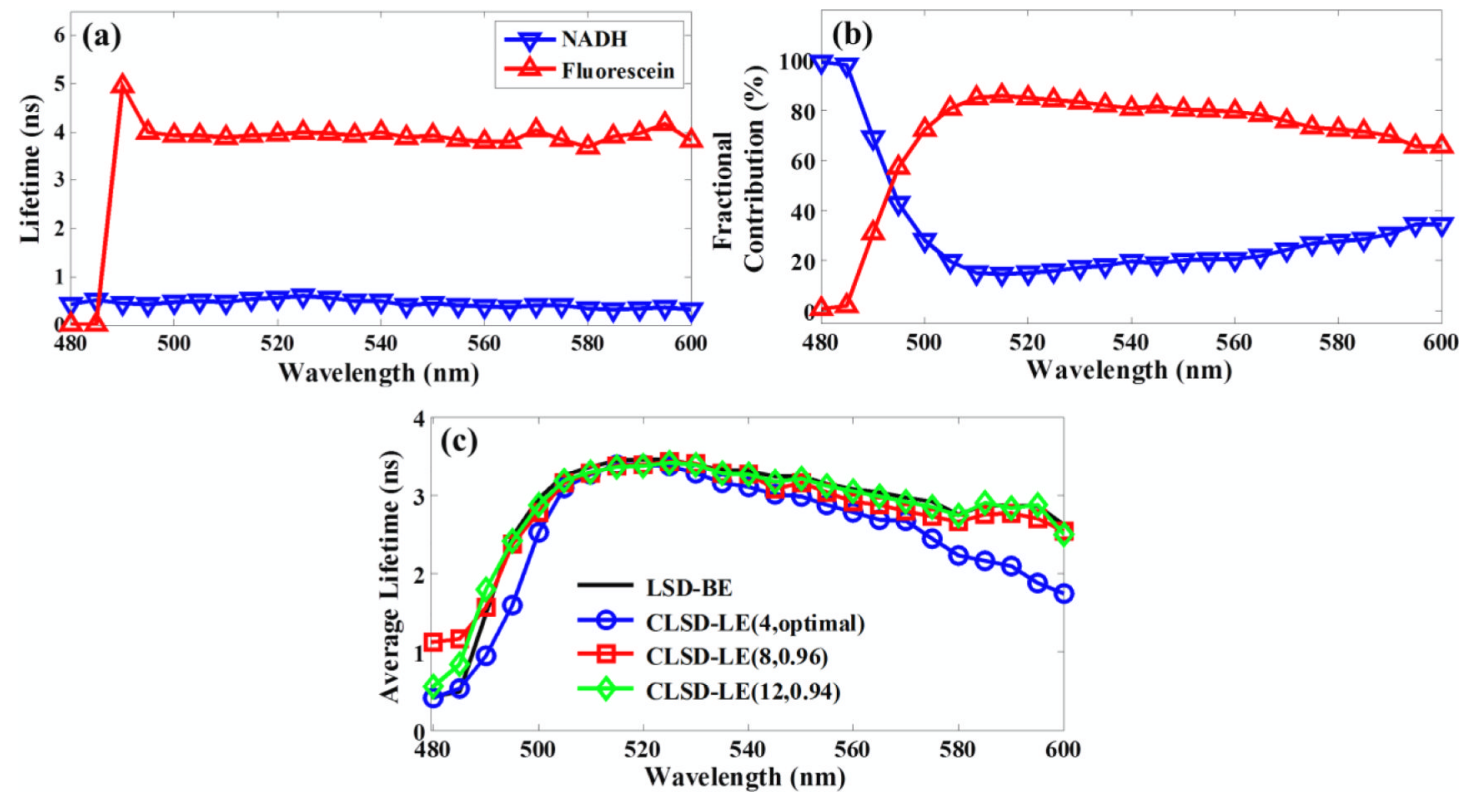

Figure 4.

Deconvolution for TRFS of a standard fluorophore mixture (2 mM NADH and $1 \mathrm{mM}$ Fluoresin, 9:1). (a) Estimated lifetimes and fractional contribution spectra for two components from LSD-BE deconvolution; (b) comparison of average lifetimes from LSDBE, CLSD-LE(4,optimal), CLSD-LE(8,0.96) and CLSD-LE(12,0.94). 

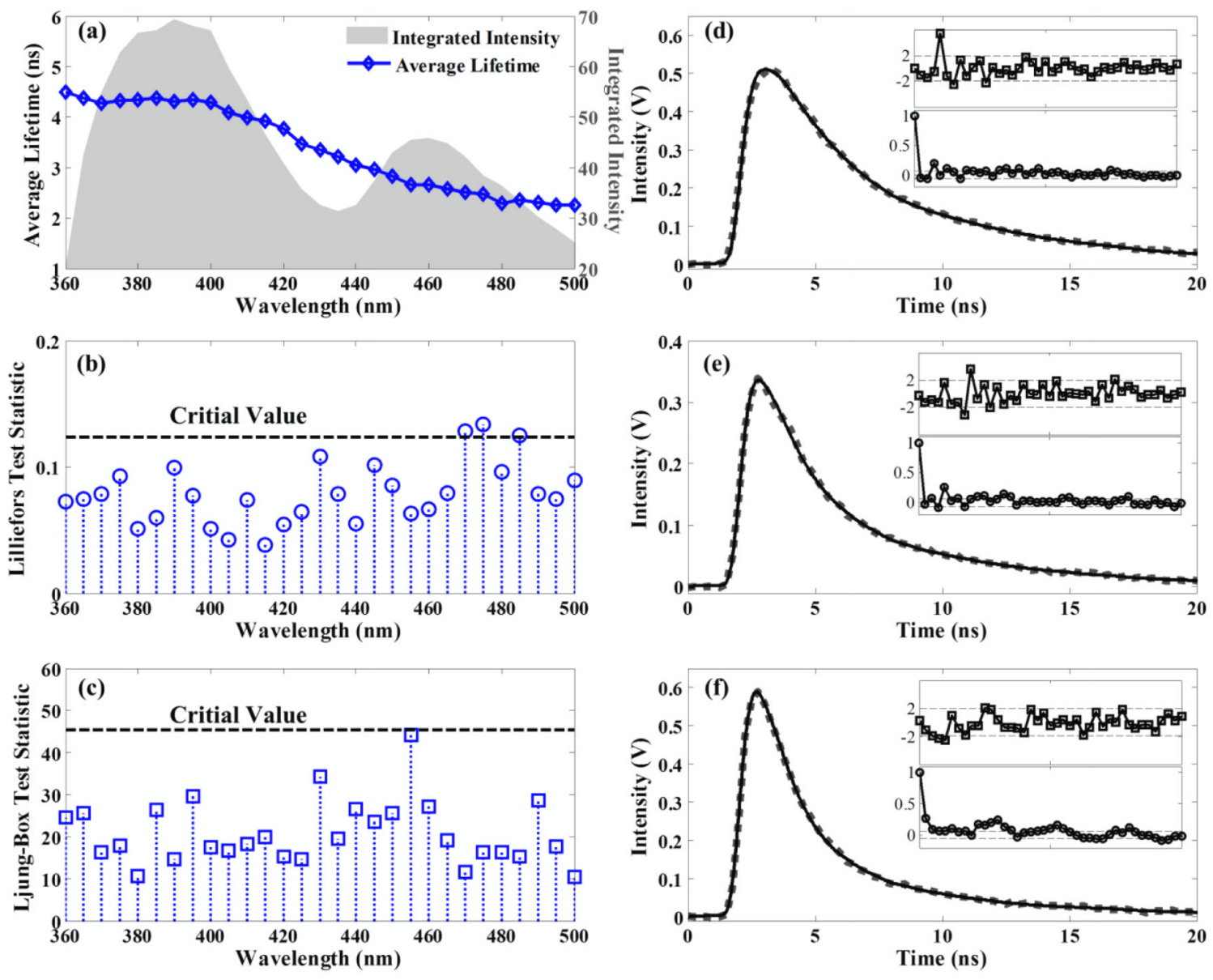

- Measured Fluorescence $\rightarrow$ Standardized Residuals
- Fitted Fluorescence $\rightarrow$ Autocorrelation Function

Figure 5.

Example of deconvolution based on CLSD-LE(8,0.96) for TRFS of carcinoma (hamster buccal pouch carcinogenesis model). (a) Integrated intensity and average lifetime spectra for 360-450 nm wavelength range; summary statistics of (b) Lilliefors and (c) Ljung-Box tests for all wavelengths and respective critical values; (d) - (f) representative deconvolution for signals measured at $380 \mathrm{~nm}, 430 \mathrm{~nm}, 460 \mathrm{~nm}$, respectively. 


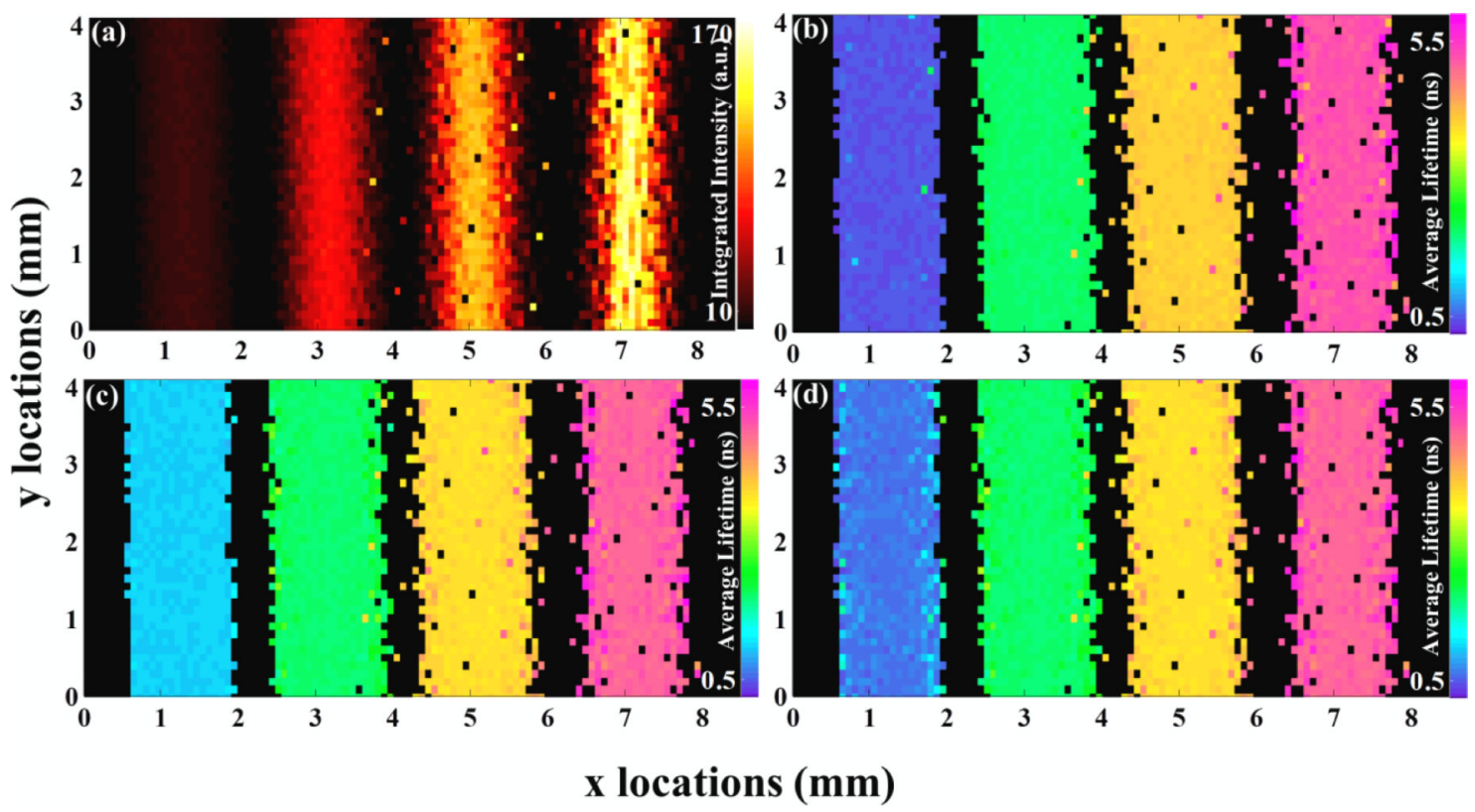

Figure 6.

LSD-BE, CLSD-LE(8,0.96) and CLSD-LE(12,0.94) for FLIM measurements of fluorophores in capillary tubes (NADH, C-1, C-120 and elastin from left to right) at $450 \mathrm{~nm}$ emission. (a) Integrated intensity map $\left(9.72 \times 4.1 \mathrm{~mm}^{2}\right)$ for scan measurement; (b), (c) and (d) estimated average lifetime images $\left(9.72 \times 4.1 \mathrm{~mm}^{2}\right)$ from LSD-BE, CLSD-LE $(8,0.96)$ and CLSD-LE(12,0.94) deconvolution respectively. 

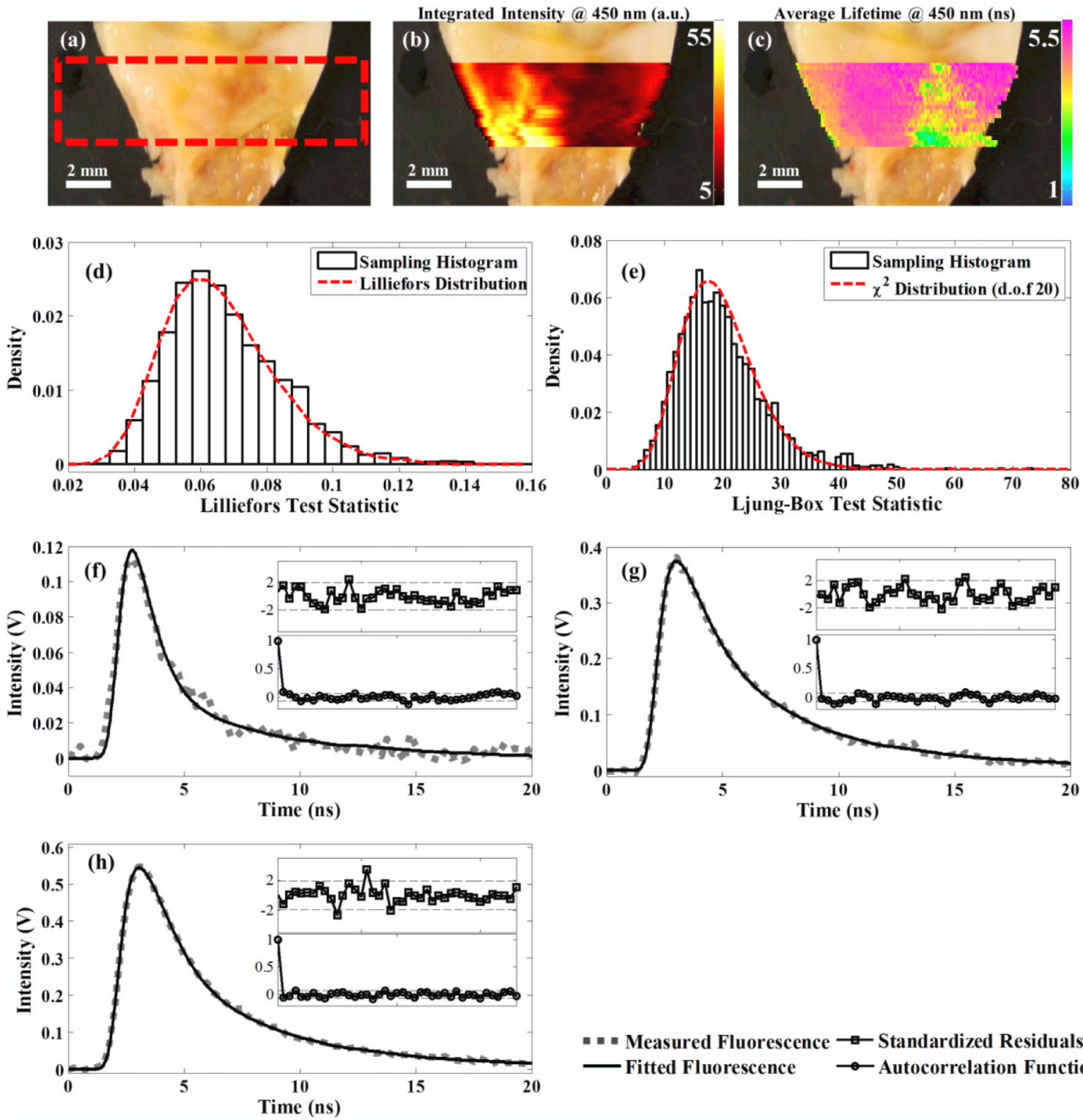

" - Measured Fluorescence $\rightarrow-$ Standardized Residuals

- Fitted Fluorescence $\rightarrow$ Autocorrelation Function

Figure 7.

Example of deconvolution based on CLSD-LE(8,0.96) for FLIM measurement of ex vivo atherosclerotic plaque. (a) Ex vivo tissue sample and scan area (red dashed box, $15 \times 4 \mathrm{~mm}^{2}$ ); (b) Integrated intensity and (c) average lifetime maps for scan measurements at $450 \mathrm{~nm}$; Sampling distributions of statistics from (d) Lilliefors and (e) Ljung-Box tests (bars) and the null distributions (red dash lines); (f), (g) and (h) Representative deconvolution for signals measured from three representative locations (lipid-rich, collagen-rich and elastin-rich respectively). 


\section{Table 1}

Upper bounds of $a$ for $L \in\{4,8,12\}$ for different truncated time intervals and sampling rates.

\begin{tabular}{|c|c|c|c|c|}
\hline & \multicolumn{2}{|c|}{ Time Interval 20 ns } & \multicolumn{2}{|c|}{ Time Interval $40 \mathrm{~ns}$} \\
\hline & $\underline{\delta t=0.05 \mathrm{~ns}}$ & $\delta t=0.125 \mathrm{~ns}$ & $\delta t=0.05 \mathrm{~ns}$ & $\delta t=0.125 \mathrm{~ns}$ \\
\hline$L=4$ & 0.96 & 0.90 & 0.98 & 0.95 \\
\hline$L=8$ & 0.92 & 0.81 & 0.96 & 0.90 \\
\hline$L=12$ & 0.88 & 0.73 & 0.94 & 0.85 \\
\hline
\end{tabular}




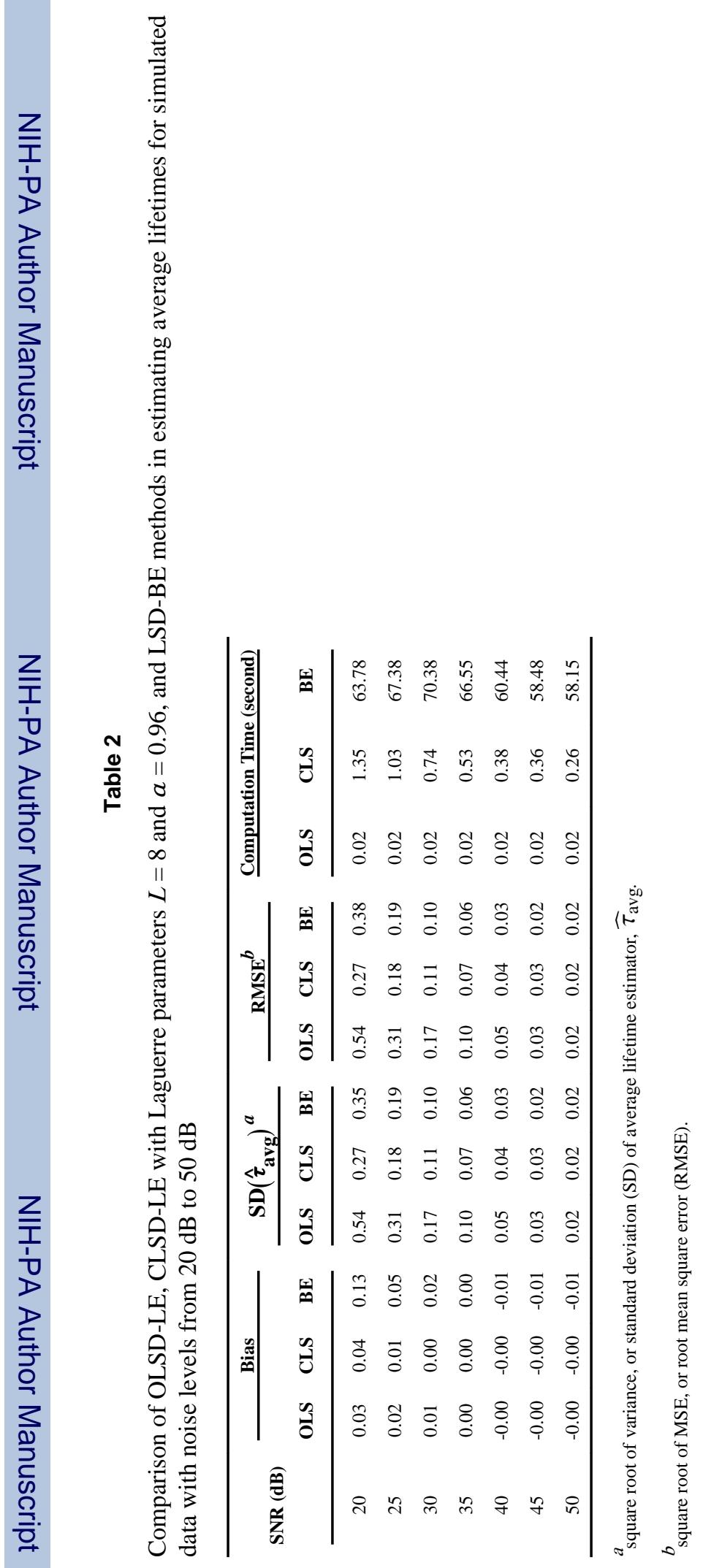

Phys Med Biol. Author manuscript; available in PMC 2013 February 21. 
สำ

2 


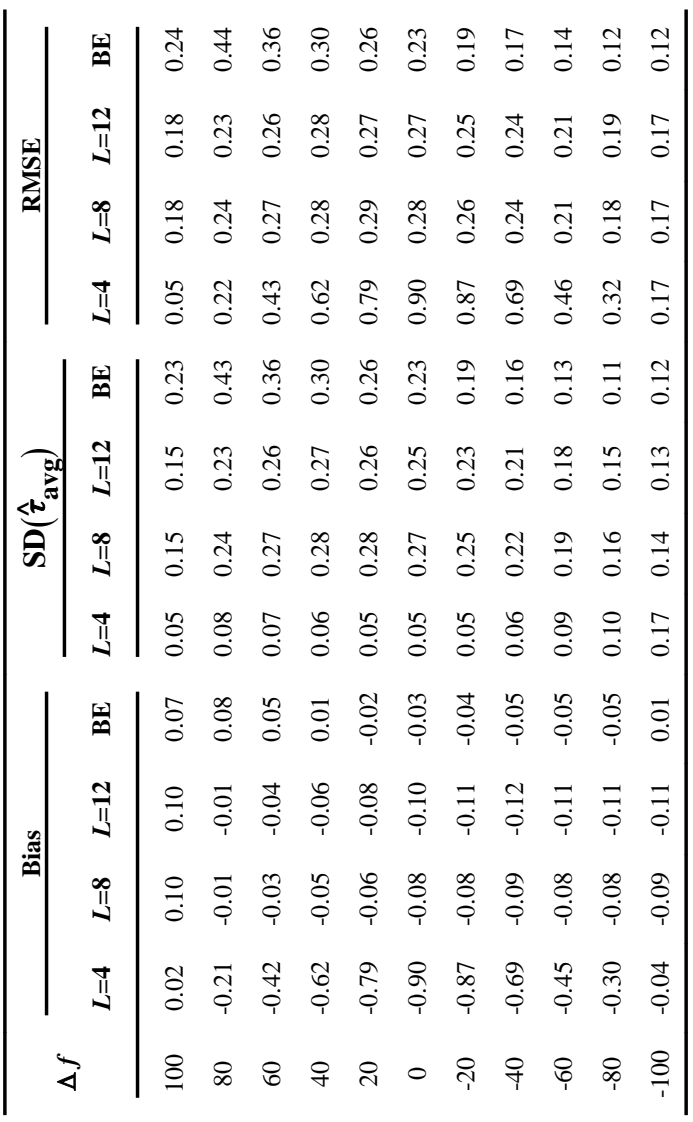


Table 5

Average lifetime values (ns) for all fluorophores from LSD-BE and CLSD-LE deconvolution.

\begin{tabular}{ccccc}
\hline & LSD-BE & CLSD-LE(8,0.96) & CLSD-LE(12,0.94) & Literature \\
\hline NADH & $0.48 \pm 0.11$ & $1.11 \pm 0.02$ & $0.68 \pm 0.14$ & $0.3 \sim 0.5$ \\
C-1 & $2.06 \pm 0.14$ & $2.08 \pm 0.18$ & $2.09 \pm 0.19$ & 2.02 \\
C-120 & $4.06 \pm 0.13$ & $3.96 \pm 0.17$ & $3.96 \pm 0.17$ & 3.85 \\
Elastin & $5.21 \pm 0.16$ & $5.09 \pm 0.15$ & $5.08 \pm 0.15$ & $5.2 \sim 7.36$ \\
\hline
\end{tabular}

